\title{
The Prevalence and Risk Factors for Dry Eye Disease among Older Adults in the City of Lodz, Poland
}

\author{
Michal S. Nowak ${ }^{1}$, Janusz Smigielski² \\ ${ }^{1}$ Department of Ophthalmology and Visual Rehabilitation, Medical University of Lodz, Lodz, Poland \\ ${ }^{2}$ Department of Geriatrics, Medical University of Lodz, Lodz, Poland \\ Email:michaelnovak@interia.pl,janusz.smigielski@umed.lodz.pl
}

Received 11 November 2015; accepted 1 February 2016; published 4 February 2016

Copyright (C) 2016 by authors and Scientific Research Publishing Inc.

This work is licensed under the Creative Commons Attribution International License (CC BY). http://creativecommons.org/licenses/by/4.0/

c) (i) Open Access

\begin{abstract}
Purpose: To estimate the prevalence and risk factors for dry eye disease (DED) in a sample population of Polish older adults. Material and methods: Cross-sectional and observational study of 1107 men and women of European Caucasian origin aged 35 - 97 years, who were interviewed and underwent detailed ophthalmic examinations. DED was defined as presence of a previous clinical diagnosis of dry eye with concomitant dry eye treatment. Results: The overall prevalence of DED in the researched population was $6.7 \%(95 \%$ CI 5.2 - 8.2). The prevalence of DED increased with age from $4.8 \%$ in age group $35-59$ years to $8.3 \%$ in group aged $\geq 60$ years. The prevalence of DED was also higher in women $8.1 \%$ than in men $4.7 \%$. In multiple logistic regression modelling with age, gender, presence of cataract surgery and glaucoma or ocular hypertension (OHT) treatment, DED was significantly associated with older age (OR 1.99, 95\% CI $1.21-3.30$ ) and with female gender (OR 1.76, 95\% CI 1.05 - 2.96). Conclusions: The prevalence of DED in our study population was comparable with the findings of other studies from Europe and the United States, with significantly higher rates among women and elderly subjects.
\end{abstract}

\section{Keywords}

Dry Eye Disease, Older Adults

\section{Introduction}

"Dry eye disease (DED) is a multifactorial disease of the tears and the ocular surface that results in symptoms of discomfort, visual disturbance, and tears film instability with potential damage to the ocular surface. It is ac- 
companied by increased osmolarity of the tear film and the inflammation of the ocular surface [1]". Dry eye is a serious health problem, with limited treatment options. DED has also a substantial economic burden on the society estimated to be 55.4 billion dollars in the United States [2] [3]. On the basis of available reports, the prevalence of DED among older adults varies from 5\% to 34\% [4] [5]. Although it is a common ocular problem, the number of studies concerning the prevalence of DED in Eastern European nations (post-soviet countries) is still very few. The aim of this study was the assessment of the prevalence and risk factors for dry eye disease in a sample population of Polish older adults.

\section{Materials and Methods}

The study design was an observational and cross-sectional. The methodology of the recruitment and subjects sampling for the current study has been described earlier. In brief: "sample size for the study was calculated with 99\% confidence, within an error bound of 5\%. The sample size requirement was 661, as calculated by

$$
n=Z^{2} / 4 d^{2},
$$

where $Z=2.57$ for $99 \%$ confidence interval and $d=0.05$ for $5 \%$ error bound. After allowing for an arbitrary $50 \%$ increase in sample size to accommodate possible inefficiencies associated with the sample design, the sample size requirement increased to 991 subjects [6] [7]”. We defined an older adult as a person aged $\geq 35$ years because in our previous reports conducted on young males in the military population we considered young adult as person aged 18 - 34 years [8] [9]. "We used simple systematic sampling to select our study population. In total 14,110 outpatients were examined in the Department of Ophthalmology and Visual Rehabilitation of the Medical University of Lodz in year 2012 and we included into the study every tenth subject aged 35 years and older [7]”. Basing on age, our study participants were divided into two groups; group I aged 35 - 59 years, and group II aged 60 years and older. All selected subjects were interviewed and underwent detailed ophthalmic examinations. Dry eye disease (DED) was defined as presence of a previous clinical diagnosis of DED with concomitant dry eye treatment (artificial tear drops or gel) like in other studies [2] [5]. This was assessed by two questions: 1) have you ever been diagnosed (by an ophthalmologist) as having dry eye disease? and 2) do you currently use any artificial tear drops or gel? Only if answer was "Yes" to both questions DED was diagnosed. For this report we used the data concerning the prevalence of cataract surgery, glaucoma and ocular hypertension (OHT) in the researched population from our recent paper [7]. All statistical analyses were performed using STATISTICA v. 10.1 PL (StatSoft Polska, Krakow, Poland) software. Prevalence rates of dry eye disease (DED) in whole population and according to the subjects' age and gender were calculated. Multiple logistic regression statistics were used to investigate the association of DED with age, gender as well as with the presence of cataract surgery and glaucoma or OHT treatment. Odds ratios (ORs) were computed, the differences were significant at p $<0.05$. All confidence intervals (CIs) were 95\% CI. The study was approved by the institutional review board of the Medical University of Lodz and was conducted in accordance with the provisions of Declaration of Helsinki for research involving human subjects.

\section{Results}

The demographic characteristics of all participants in the study and statistical analyses are presented in Table 1. A total of 1107 subjects aged $\geq 35$ years were successfully enumerated and included into the study. All of them were of European Caucasian origin. According to 2011 national census, they were a fair representation of the population of the city of Lodz in terms of sex distribution (statistical analysis-chi square test: $\chi^{2}=3.64$, p $>0.05$ ) and socioeconomic status [10]. The city of Lodz is the second largest city in Poland and consists of seven hundred forty thousand inhabitants (2011 national census), mostly of middle socioeconomic level [10]. The mean age of our study participants was $60.4 \pm 7.1$ years (range, 35 to 97 years). There were 642 women (58.0\%) and 465 men (42.0\%) and they were divided into two age groups: 520 (47.0\%) subjects were aged 35 - 59 years, and 587 (53.0\%) subjects were aged $\geq 60$ years. The overall prevalence of dry eye disease in the researched population (diagnosed by clinician with concomitant dry eye treatment) was 6.7\% (95\% confidence interval [CI] 5.2 8.2). The prevalence of DED increased with age from 4.8\% (95\% CI 3.0 - 6.6) in age group 35 - 59 years to $8.3 \%$ (95\% CI $6.1-10.6)$ in group aged $\geq 60$ years (Figure 1 ). The prevalence of dry eye was also higher in women 8.1\% (95\% CI 6.0 - 10.2) than in men 4.7\% (95\% CI 2.8 - 6.7) (Figure 2). Statistical analysis revealed that the differences between prevalence rates of dry eye disease (DED) among genders and particular age groups were 
Table 1. The demographic characteristics of examined group.

\begin{tabular}{|c|c|c|c|c|c|c|c|c|}
\hline Examined group & Number of subjects: n (\%) & Min & $\operatorname{Max}$ & Mean & Med & Std. dev. & Men & Women \\
\hline All & 1107 (100\%) & 35.0 & 97.0 & 60.4 & 61.0 & 12.8 & 465 (100\%) & $642(100 \%)$ \\
\hline 35 - 59 years & $520(47.0 \%)$ & 35.0 & 59.0 & 49.3 & 50.0 & 7.1 & 230 (49.5\%) & $290(45.2 \%)$ \\
\hline$\geq 60$ years & $587(53.0 \%)$ & 60.0 & 97.0 & 70.1 & 69.0 & 7.8 & 235 (50.5\%) & $352(54.8 \%)$ \\
\hline Examined group & Number of subjects: n (\%) & Min & $\operatorname{Max}$ & Mean & Med & Std. dev. & 35 - 59 years & $\geq 60$ years \\
\hline All & 1107 (100\%) & 35.0 & 97.0 & 60.4 & 61.0 & 12.8 & $520(100 \%)$ & 587 (100\%) \\
\hline Men & $465(42.0 \%)$ & 35.0 & 97.0 & 59.8 & 60.0 & 14.1 & $230(44.2 \%)$ & $235(40.0 \%)$ \\
\hline Women & $642(58.0 \%)$ & 35.0 & 93.0 & 60.7 & 61.0 & 11.7 & 290 (55.8\%) & $352(60.0 \%)$ \\
\hline
\end{tabular}

$\chi^{2}$ test $\mathrm{p}=0.158$.

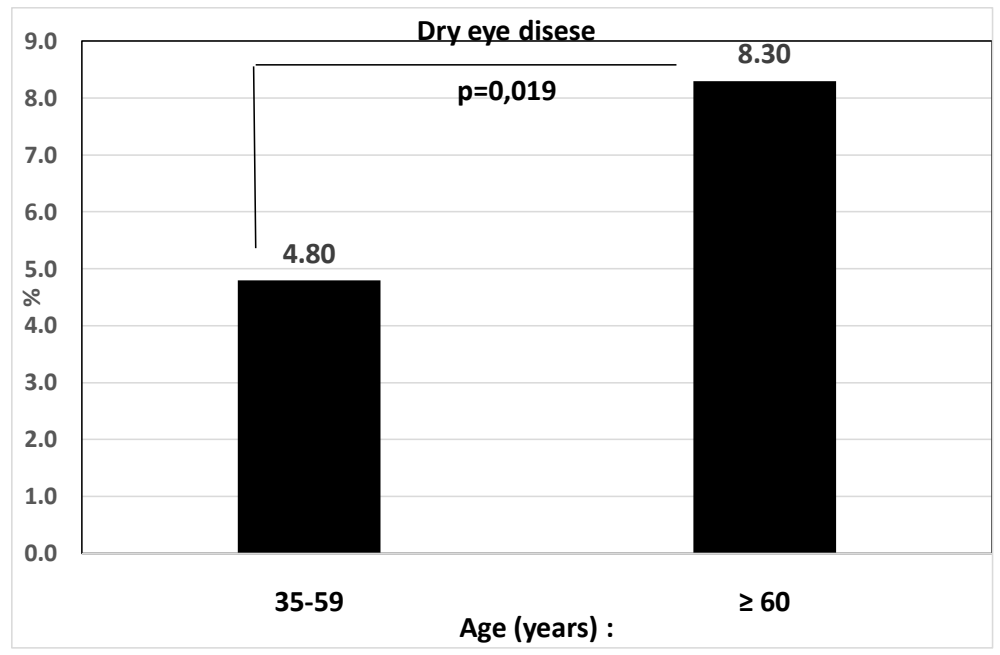

Figure 1. The prevalence of dry eye disease as a function of age.

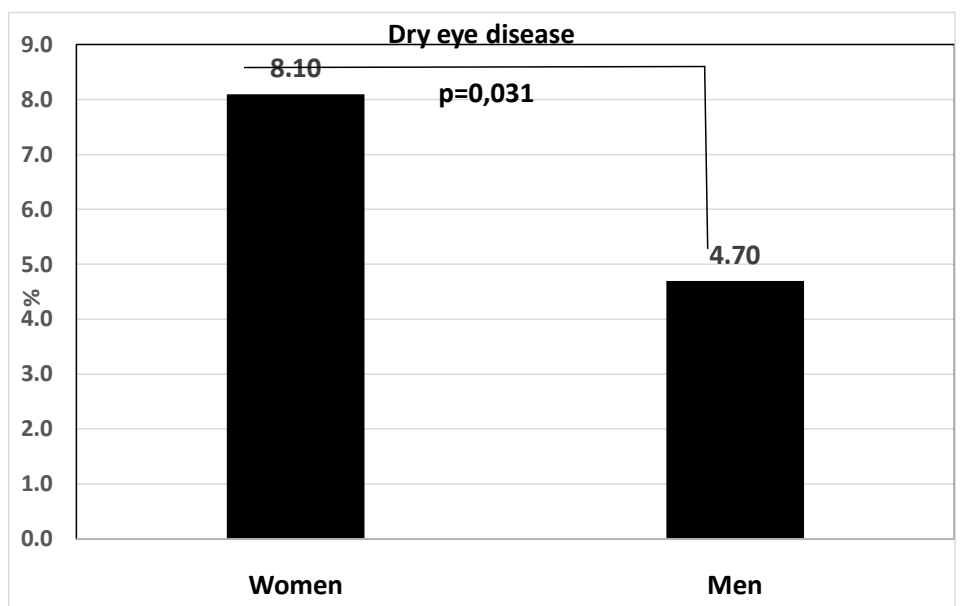

Figure 2. The prevalence of dry eye disease among genders. 
statistically significant $\left(\chi^{2}\right.$ test $\mathrm{p}=0.031$ and $\mathrm{p}=0.019$ respectively). As reported earlier 8.04\% (95\% CI 6.44 9.64) of all participants in the study had cataract surgery in either eye. Various types of glaucoma were diagnosed in 5.51\% (95\% CI 4.17 - 6.85) of subjects and 2.62\% (95\% CI 1.68 - 3.56) had ocular hypertension (OHT). Multivariate logistic regression model was constructed to analyze the risk factors for dry eye in this population. In multiple logistic regression modelling with age, gender, presence of cataract surgery and glaucoma or ocular hypertension (OHT) treatment, dry eye disease (DED) was significantly associated with older age (OR 1.99, 95\% CI 1.21 - 3.30) and with female gender (OR 1.76, 95\% CI 1.05 - 2.96). However no association was found between DED and the presence of cataract surgery and glaucoma or OHT treatment.

\section{Discussion}

The current study provides for the first time reliable data concerning the prevalence and risk factors for dry eye disease (DED) in a large unselected population of Polish citizens aged 35 years and older. Overall the prevalence of DED in the examined population was rather low (6.7\%) and was higher in women (8.1\%) than in men (4.7\%). The prevalence of DED was also significantly higher in the age group $\geq 60$ years. Direct comparison of our results to the results of other studies on DED is limited due to differences in study design and population sampling. The major limitation is the fact that the presence of DED was determined by subject self-reported history of dry eye. We did not perform any diagnostic tests i.e. Schirmer test, tear break-up time measurement (TBUT), rose bengal staining or fluoresceine staining to confirm the clinical diagnosis of DED. However our prevalence rate of dry eye was closer to the results obtained in a British female cohort study, in which they used the same definition of DED and found that $9.6 \%$ of women had a DED diagnosis with concomitant dry eye treatment [2]. In addition our results were similar to those reported in large epidemiological studies from the United States (Women's Health Study and Physician's Health Study), which indicated that the prevalence of symptomatic dry eye disease is about $7 \%$ in women and $4 \%$ of men over the age of 50 years [4] [11] [12]. Although previous studies identified several risk factors for the development of DED like aging, gender, hormonal changes, contact lens wear, certain medications and surgical procedures [4], in the present study multiple regression analysis showed that DED was only associated with older age and with female gender.

\section{Conclusion}

In conclusion, the prevalence of DED in our study population was comparable with the findings of other studies from Europe and the United States, with significantly higher rates among women and elderly subjects. Further studies are needed to confirm some of the previously identified risk factors.

\section{Conflict of Interests}

The authors declare that there is no conflict of interests regarding the publication of this paper.

\section{References}

[1] (2007) The Definition and Classification of Dry Eye Disease: Report of the Definition and Classification Subcommittee of the International Dry Eye Workshop (2007). The Ocular Surface, 5, 75-92. http://dx.doi.org/10.1016/S1542-0124(12)70081-2

[2] Vehof, J., Kozareva, D., Hysi, P.G. and Hammond, C.J. (2014) Prevalence and Risk Factors of Dry Eye Disease in a British Female Cohort. British Journal of Ophthalmology, 98, 1712-1717. http://dx.doi.org/10.1136/bjophthalmol-2014-305201

[3] Yu, J., Asche, C.V. and Fairchild, C.J. (2011) The Economic Burden of Dry Eye Disease in the United States: A Decision Tree Analysis. Cornea, 30, 379-387. http://dx.doi.org/10.1097/ICO.0b013e3181f7f363

[4] Gayton, J.L. (2009) Etiology, Prevalence, and Treatment of Dry Eye Disease. Clinical Ophthalmology, 3, 405-412. http://dx.doi.org/10.2147/OPTH.S5555

[5] Galor, A., Feuer, W., Lee, D.J., Florez, H., Carter, D., Pouyeh, B., Prunty, W.J. and Perez, V.L. (2011) Prevalence and Risk Factors of Dry Syndrome in a United States Veterans Affairs Population. American Journal of Ophthalmology, 152, 377-384. http://dx.doi.org/10.1016/j.ajo.2011.02.026

[6] Nowak, M.S. and Smigielski, J. (2015) The Prevalence and Causes of Visual Impairment and Blindness among Older Adults in the City of Lodz, Poland. Medicine (Baltimore), 94, e505. http://dx.doi.org/10.1097/MD.0000000000000505 
[7] Nowak, M.S. and Smigielski, J. (2015) The Prevalence of Age-Related Eye Diseases and Cataract Surgery among Older Adults in the City Lodz, Poland. Journal of Ophthalmology, Article ID: 605814. http://dx.doi.org/10.1155/2015/605814

[8] Nowak, M.S., Goś, R., Jurowski, P. and Śmigielski, J. (2009) Correctable and Non-Correctable Visual Impairment among Young Males: A 12-Year Prevalence Study of the Military Service in Poland. Ophthalmic and Physiological Optics, 29, 443-448. http://dx.doi.org/10.1111/j.1475-1313.2008.00628.x

[9] Nowak, M.S., Jurowski, P., Goś, R. and Śmigielski, J. (2010) Ocular Findings among Young Men: A 12 Year Prevalence Study of Military Service in Poland. Acta Ophthalmologica, 88, 535-540. http://dx.doi.org/10.1111/j.1755-3768.2008.01476.x

[10] The National Census of Population and Housing 1 April - 30 June 2011. Zakład Wydawnictw Statystycznych. Warszawa 2013. http://www.stat.gov.pl/gus/nsp

[11] Schaumberg, D.A., Sullivan, D.A., Buring, J.E. and Dana, M.R. (2003) Prevalence of Dry Eye Syndrome among US Women. American Journal of Ophthalmology, 136, 318-326. http://dx.doi.org/10.1016/S0002-9394(03)00218-6

[12] Schaumberg, D.A., Dana, R., Buring, J.E. and Sullivan, D.A. (2009) Prevalence of Dry Eye Disease among US Men: Estimates from the Physicians' Health Studies. Archives of Ophthalmology, 127, 763-768.

http://dx.doi.org/10.1001/archophthalmol.2009.103 